DOI: $10.15593 / 2224-9982 / 2017.50 .13$

УДК 629.735.03:62/. 43.056

\author{
А.Д. Нугуманов ${ }^{1,2}$, А.М. Сипатов ${ }^{1,2}$, В.А. Назукин ${ }^{1}$ \\ ${ }^{1}$ АО «ОДК-Авиадвигатель», Пермь, Россия \\ ${ }^{2}$ Пермский национальный исследовательский политехнический университет, Пермь, Россия
}

\author{
ИСПОЛЬЗОВАНИЕ МИРОВОГО ОПЫТА РЕГУЛИРОВАНИЯ \\ ГАЗОТУРБИННЫХ ДВИГАТЕЛЕЙ (ГТД) С МАЛОЭМИССИОННОЙ \\ КАМЕРОЙ СГОРАНИЯ (МЭКС) ПО РЕЖИМАМ МОЩНОСТИ \\ И В КЛИМАТИЧЕСКОМ ДИАПАЗОНЕ ПРИ СОЗДАНИИ МЭКС ДЛЯ ГТД \\ РАЗРАБОТКИ АО «ОДК-АВИАДВИГАТЕЛЬ»
}

\begin{abstract}
Выполнен анализ мирового опыта регулирования газотурбинных двигателей (ГТД) с малоэмиссионными камерами сгорания, работающими по технологии сжигания «бедных» заранее перемешанных топливовоздушных смесей (Lean Premixed Prevaporized (LPP)), которая считается на сегодняшний день наиболее перспективной для стационарных ГТУ. Разработкой этой технологии за рубежом ведущие фирмы мира занимаются уже более тридцати лет. Переход на эту технологию позволил снизить уровень эмиссии $\mathrm{NO}_{x}$ и $\mathrm{CO}$ более чем на порядок, доведя эмиссию $\mathrm{NO}_{x}$ в лучших образцах малоэмиссионных камер сгорания (МЭКС) ГТУ до 9-15 ppm, что, безусловно, можно считать переворотом в решении экологических проблем. Одним из сложнейших вопросов в данной технологии является поддержание постоянной температуры в зоне горения в широком диапазоне мощности при различных климатических условиях. Рассмотрены основные способы поддержания постоянной температуры в зоне горения применительно к современным ГтД зарубежных фирм, а именно: различные сбросы и перепуски воздуха из/внутри камеры сгорания; многоколлекторная стадийная подача топлива; поворотные лопатки входного направляющего аппарата компрессора. Представленные материалы формируют рекомендации по выбору конструктивных схем регулирования ГТД с МЭКС «бедного» типа. На основании представленного материала выполнен анализ возможности применения основных способов регулирования ГТД разработки АО «ОДК-Авиадвигатель» с МЭКС.

Ключевые слова: малоэмиссионная камера сгорания, оксид азота, монооксид углерода, коэффициент избытка воздуха, перепуск воздуха, многоколлекторная подача топлива, поворотный входной направляющий аппарат, газотурбинная установка.
\end{abstract}

\author{
A.D. Nugumanov ${ }^{1,2}$, A.M. Sipatov ${ }^{1,2}$, V.A. Nazukin ${ }^{1}$ \\ ${ }^{1}$ UEC-Aviadvigatel JSC, Perm, Russian Federation \\ ${ }^{2}$ Perm National Research Politechnic University, Perm, Russian Federation
}

\title{
APPLICATION OF WORLD PRACTICES IN DLN GAS TURBINE CONTROL OVER THE POWER AND CLIMATIC RANGE DURING DEVELOPMENT OF LOW EMISSION COMBUSTOR DESIGNED BY UEC-AVIADVIGATLE JSC
}

This work analyses the world experience of regulating the DLN gas turbines using Lean Premixed Prevaporized (LPP) technology which is currently the most advanced one for the frame gas turbine application. World benchmark companies have been developing this technology for over 30 years. The transition to this technology allowed reduction of $\mathrm{NO}_{x}$ and $\mathrm{CO}$ emissions by more than an order of magnitude bringing $\mathrm{NO}_{x}$ in the best prototypes of gas turbine DLNs to 9-15 ppm which certainly can be considered a coup in solving environmental problems. One of the most difficult issues in this technology is how to maintain a temperature in the combustion zone constant over a wide power range under different climatic conditions. This work discusses basic methods to maintain a constant temperature in the combustion zone referring to the current gas turbine engines of world companies, i.e. (1) various air bleeds from/within the combustor; (2) multi-manifold staged fuel supply; (3) variable inlet guide vanes (VIGV).

The presented materials formulate recommendations on selection of control diagrams for DLN gas turbines. Based on the presented material, this work analyses possibility of applying the main methods to regulate gas turbine engine with Low Emission Combustor developed by UEC-Aviadvigatel JSC.

Keywords: low emission combustor, nitrogen oxide, carbon monoxide, air-fuel ratio, air bleed, multi-manifold fuel supply, variable geometry igv, gas turbine unit. 


\section{Введение}

Интенсивное развитие энергетики в мире привело к значительному загрязнению атмосферы и окружающей среды вредными выбросами продуктов сгорания. В связи с этим снижение выбросов вредных веществ стало наиболее актуальной задачей. Ужесточение международных и отечественных требований по снижению вредных выбросов $\mathrm{NO}_{x}$ и $\mathrm{CO}$ до $25 \mathrm{ppm}$ привело к необходимости разработки новых малоэмиссионных технологий и использованию природного газа в качестве приоритетного вида топлива. На сегодняшний день технология Lean Premixed Combustion (LP) является наиболее перспективной для стационарных газотурбинных установок (ГТУ). Рассматриваемая технология за рубежом совершенствуется более тридцати лет. Переход на LP-технологию позволил значительно снизить уровень эмиссии $\mathrm{NO}_{x}$ и $\mathrm{CO}$, доведя эмиссию в лучших образцах малоэмиссионных камер сгорания (МЭКС) ГТУ до 9-15 ppm, что, безусловно, можно считать переворотом в решении экологических проблем [1].

Вместе с тем многолетний опыт зарубежных фирм по внедрению технологии LP показал, что для ее реализации необходимо решить ряд сложных проблем фундаментального, научного и технического характера - таких, как: создание эффективных и компактных смесителей с низким уровнем пульсации концентраций топлива; устранение причин проскока пламени из жаровой трубы в фронтовое устройство; расширение границ «бедного» срыва пламени; существенное снижение амплитуды пульсаций давления газа в камере; реализация чисто конвективной системы охлаждения стенок жаровой трубы; разработка систем регулирования параметров, позволяющих поддерживать в широком диапазоне мощностей ГТУ условия малоэмиссионного сжигания топлива [2-4].

\section{Проблемы организации устойчивой работы МЭКС в ГТУ}

«Бедные» МЭКС имеют очень узкий диапазон устойчивой работы по коэффициенту избытка воздуха - альфа. Данный коэффициент может очень сильно меняться при изменении температуры окружающего воздуха $T_{\text {н }}$ Как правило, в таких МЭКС зона реакции настроена максимально «горячо» при температуре МCA $\left(+15{ }^{\circ} \mathrm{C}\right)$, а с понижением или повышением, $T_{\text {н }}$ происходит ее забеднение, сопровождающееся ростом выбросов СО, появлением виброгорения и падением полноты сгорания, особенно на низких режимах работы ГТУ. Для регулирования МЭКС при изменении температуры окружающего воздуха используются те же механизмы, что и для регулирования при дросселировании, поскольку и в том, и в другом случае необходимо поддерживать постоянную температуру в зоне горения, а именно:

1. Различные сбросы и перепуски воздуха из/внутри камеры сгорания.

2. Многоколлекторная стадийная подача топлива.

3. Поворотные лопатки входного направляющего аппарата (ВНА) компрессора.

Однако, несмотря на развитое регулирование, ГТД с МЭКС имеют ограниченный малоэмиссионный режим работы по мощности и температуре окружающего воздуха. В таблице приведена информация о диапазонах работы различных ГТД, согласно презентации компании Trans Canada, являющейся крупнейшим оператором газопроводов в Канаде.

Диапазоны работы различных ГТД в малоэмиссионном режиме

\begin{tabular}{|l|c|c|c|c|c|c|}
\hline Производитель & ГТД & $\mathrm{NO}_{x}, \mathrm{ppm}$ & $\mathrm{CO}, \mathrm{ppm}$ & Мин. нагрузка, $\%$ & Мин. $T_{\mathrm{H}},{ }^{\circ} \mathrm{C}$ & Макс. $T_{\mathrm{H}},{ }^{\circ} \mathrm{C}$ \\
\hline \multirow{3}{*}{$\mathrm{GE}$} & $\mathrm{LM} 1600$ & 25 & 25 & 75 & $-9,4$ & 37,8 \\
\cline { 2 - 8 } & $\mathrm{LM} 2500$ & 25 & 25 & 55 & $\mathrm{H} /$ д & $\mathrm{H} /$ д \\
\cline { 2 - 8 } & $\mathrm{LM6000}$ & 25 & 25 & 55 & $\mathrm{H} /$ д & $\mathrm{H} /$ д \\
\hline Siemens & $\mathrm{SGT400}$ & 15 & 10 & 50 & -20 & 30 \\
\hline Rolls-Royce & $\mathrm{RB} 11$ & 25 & 25 & 78 & -30 & 40 \\
\hline Solar & SoloNO $_{x}$ & 15 & 25 & 50 & -20 & 48,9 \\
\hline
\end{tabular}


К настоящему времени эти данные уже могли устареть, но из них видно, что ГТД с МЭКС имеют ограниченный по температуре режим работы с низкой эмиссией. Ниже будут рассмотрены основные способы регулирования, используемые различными производителями ГТД.

\section{Анализ мирового опыта регулирования ГТД с МЭКС}

В авиапроизводных ГТД компании Rolls-Royce (Industrial RB211, Industrial Trent), которые в настоящее время выпускаются компанией Siemens, реализовано стадийное подключение топливных коллекторов. В двигателе RB211 две стадии, разнесенные по длине жаровой трубы, в двигателе Trent - три стадии, схемы камер сгорания представлены на рис. 1.
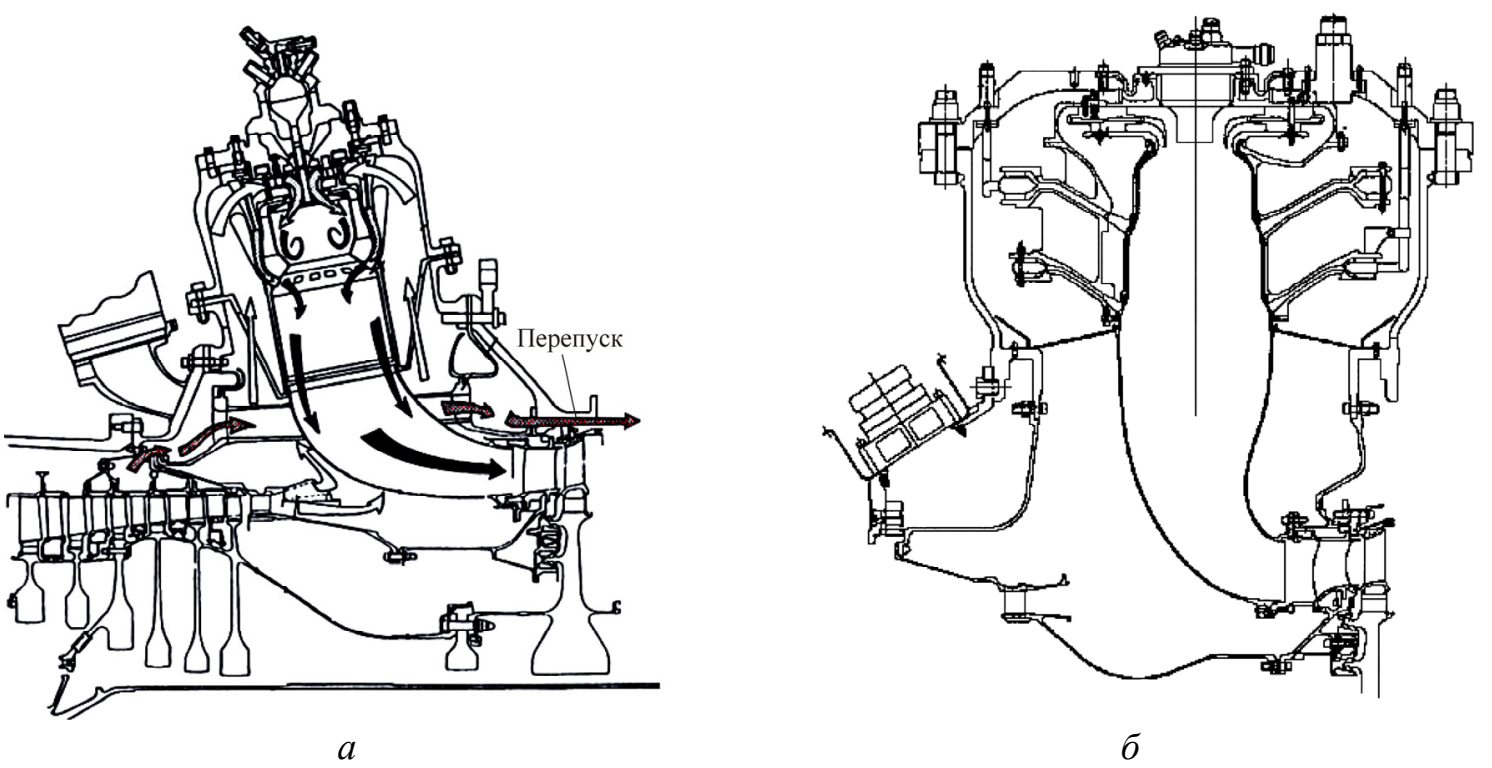

Рис. 1. Камеры сгорания двигателей Industrial RB211 (a) и Trent (б)

Согласно опубликованным данным [5], использование стадийного подключения коллекторов и управление подачей топлива в каждую из зон горения позволяет работать в широком диапазоне мощности и температуры окружающей среды без изменяемой геометрии и перепусков. Тем не менее в двигателе RB211 присутствует перепуск воздуха из компрессора за турбину высокого давления (см. рис. 1). В статье, посвященной камере сгорания Industrial Trent [6], также было упомянуто, что контролируется расход воздуха в жаровую трубу, чтобы поддерживать температуру в зоне горения, а оставшийся воздух сбрасывается ниже по потоку от камеры сгорания.

ГТУ на базе RB211 предлагается с двумя вариантами камер сгорания (КС): традиционной и малоэмиссионной, при этом ГТУ с МЭКС имеет КПД на $0,2 \%$ меньше, чем с традиционной (37,5 против 37,7 \%), что, вероятно, связано с увеличенными потерями полного давления.

В ГТУ Titan 130 компании Solar используется система управления расходом воздуха во фронт жаровой трубы (рис. 2). В зависимости от параметров двигателя, которые, в свою очередь, зависят от температуры наружного воздуха, автоматика регулирует расход воздуха во фронтовое устройство с помощью регулирующего клапана, чтобы поддерживать во фронте жаровой трубы требуемую температуру [7]. В случае необходимости лишний воздух сбрасывается в выхлопную шахту ГТУ через клапан перепуска. В систему управления также включено непосредственное измерение эмиссии $\mathrm{CO}$, на основании которого настраивается расход воздуха в жаровую трубу. Данная регулировка позволяет обеспечивать эмиссию $\mathrm{NO}_{x}$ и $\mathrm{CO}$ ниже $25 \mathrm{ppm}$ от 50 до $100 \%$ мощности при температуре окружающего воздуха до $-18{ }^{\circ} \mathrm{C}$. 


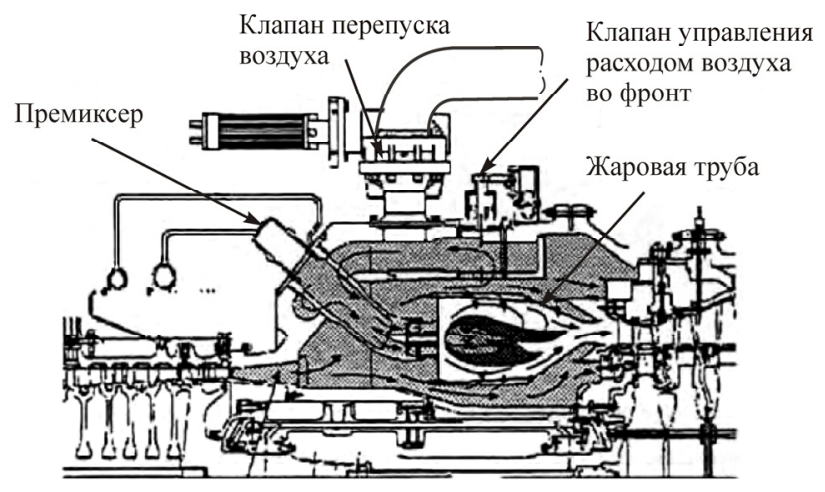

Рис. 2. Камера сгорания двигателя Titan 130

В промышленных двигателях компании GE с камерой сгорания DLN-1 наряду с поворотными лопатками ВНА используется система перепуска воздуха на вход двигателя [8]. Поворотные лопатки ВНА позволяют снижать режим работы ГТУ примерно до 80 \% от номинального с сохранением приемлемого уровня эмиссии, затем используется перепуск воздуха, согласно

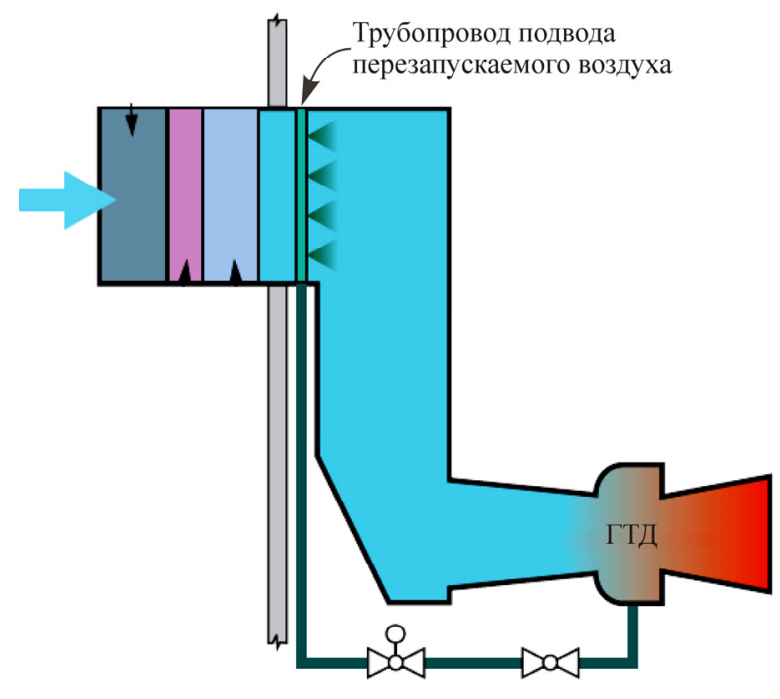

Рис. 3. Схема перепуска воздуха на вход в двигатель в ГТУ с КС DLN-1

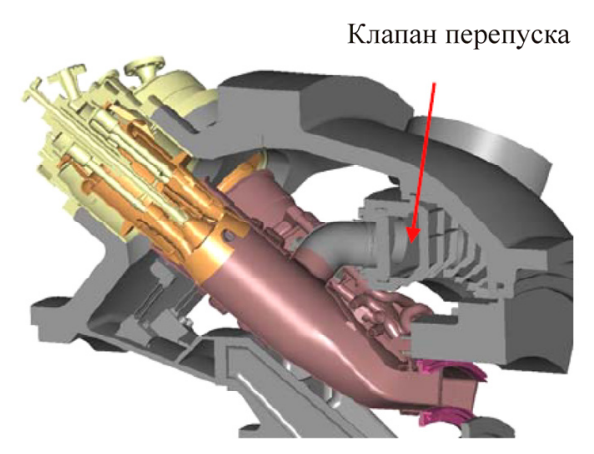

Рис. 4. Камера сгорания ГТУ $G$-класса фирмы МHI схеме, представленной на рис. 3 , который позволяет сохранять малоэмиссионный режим работы до 50-60 \%, а при определенных внешних условиях и до $40 \%$. Подогрев поступающего воздуха за счет его смешивания с горячим воздухом из-за компрессора позволяет избежать проблемы обледенения.

Одной из последних модификаций камеры сгорания DLN-1 является внедрение технологии Late Fuel Staging (LFS), предполагающей подачу $12 \%$ топлива через основные отверстия в газосборнике. Для данного решения приводятся следующие цифры: снижение нагрузки до $35 \%$ с сохранением низкой эмиссии при перепуске воздуха на вход или до $75 \%$ без перепуска [9].

В ГТУ фирмы Mitsubishi Heavy Industries для поддержания необходимой температуры в жаровой трубе применяются поворотные лопатки ВНА и перепуск воздуха в газосборник с использованием регулировочного клапана (рис. 4) [10]. Высокой неравномерности поля температур, возможно, удается избежать за счет того, что перепускаемый воздух подается в средней части жаровой трубы на значительном удалении от лопаток соплового аппарата первой ступени.

Стоит отметить тот факт, что в следующем поколении ГТУ (J-класс) клапан перепуска воздуха отсутствует. Характеристики на режимах частичной нагрузки обеспечиваются за счет поворотного ВНА и НА первых трех ступеней [11]. 
В ГТУ малой мощности фирмы Siemens Industrial Turbomachinery без регулирования обеспечивается работа в малоэмиссионном режиме в диапазоне мощности от 70 до $100 \%$ при температуре окружающего воздуха от -20 до $+40{ }^{\circ} \mathrm{C}$. Для его расширения помимо поворотных лопаток ВНА используется перепуск воздуха из-за компрессора в выхлопную шахту или на вход двигателя (рис. 5), что приводит к росту эмиссии оксидов азота, но позволяет расширить диапазон работы с приемлемой эмиссией СО [12].
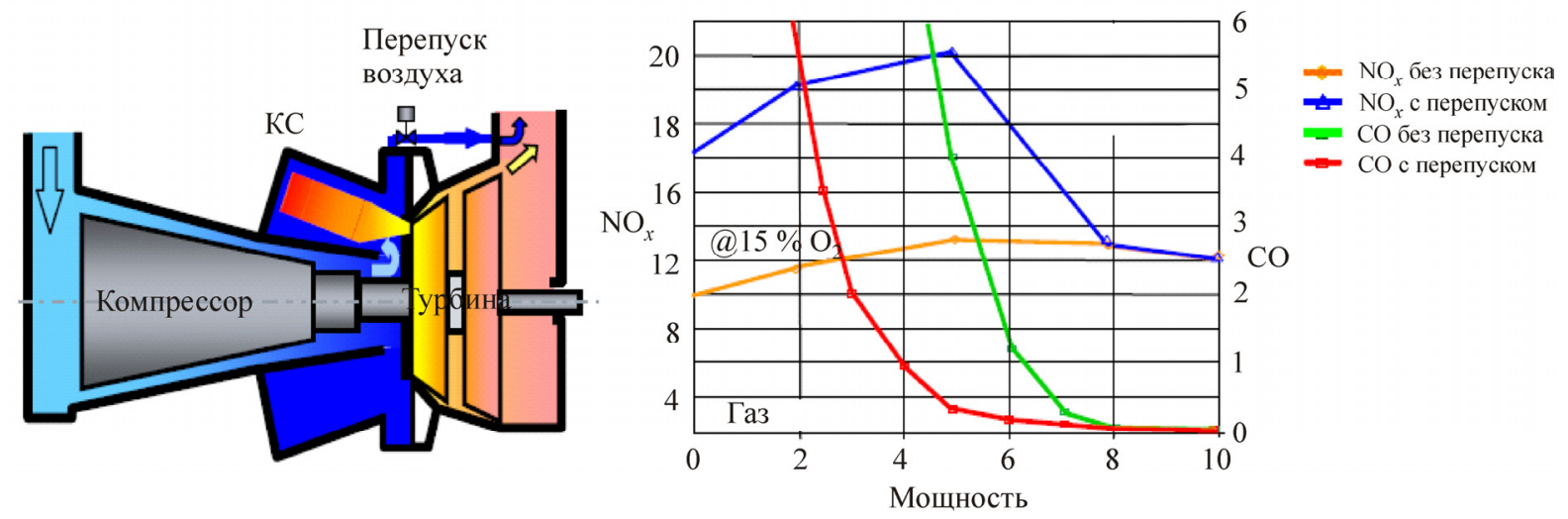

Рис. 5. Схема перепуска воздуха в выхлопную шахту в ГTУ SGT-400 (слева) и эмиссионные характеристики (справа)

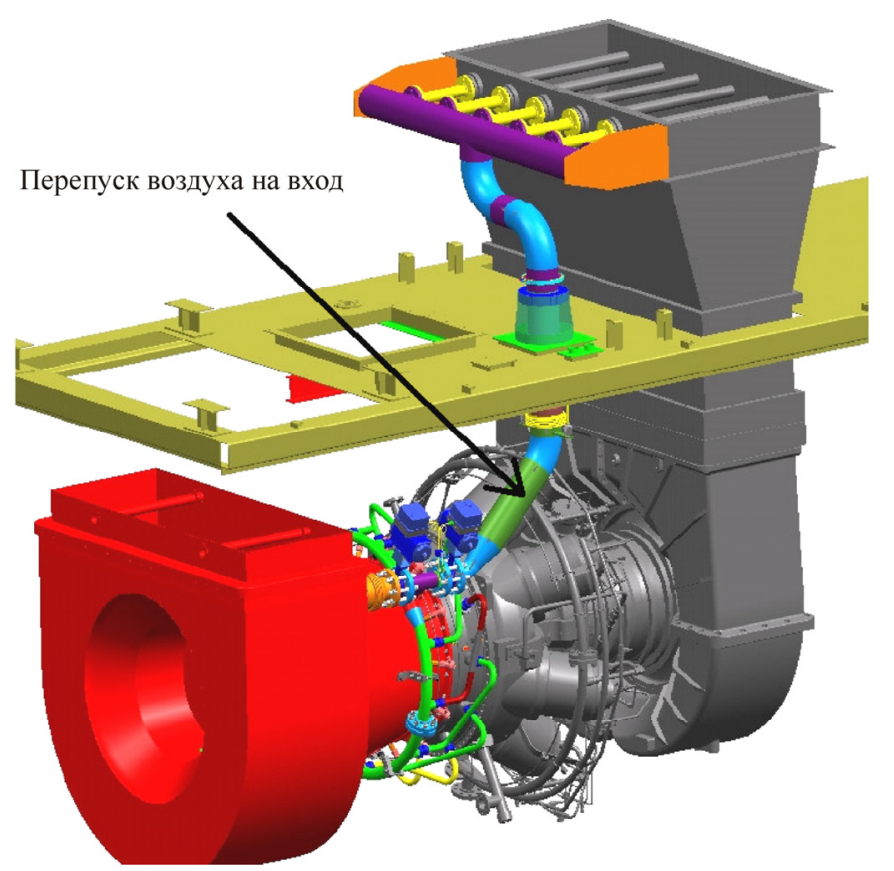

Рис. 6. Перепуск воздуха на вход двигателя SGT-400

В ГТУ большой мощности компании Siemens в настоящее время предлагается многомодульная камера сгорания с ультранизкой эмиссией $\mathrm{NO}_{x}$ [13], которая благодаря регулируемому ВНА, пятиколлекторной системе подачи топлива и системе управления расходом воздуха обеспечивает эмиссию $\mathrm{NO}_{x}$ и $\mathrm{CO}$ ниже $10 \mathrm{ppm}$ в диапазоне мощности от 30 до $100 \%$ в широком диапазоне условий окружающей среды. Точные цифры для температуры воздуха и величины перепусков не приводятся, однако, согласно приведенной схеме [14], можно увидеть, что предусмотрен перепуск воздуха из-за последних ступеней компрессора и из корпуса камеры сгорания на вход в двигатель, на выход и к промежуточным ступеням турбины (рис. 7). 


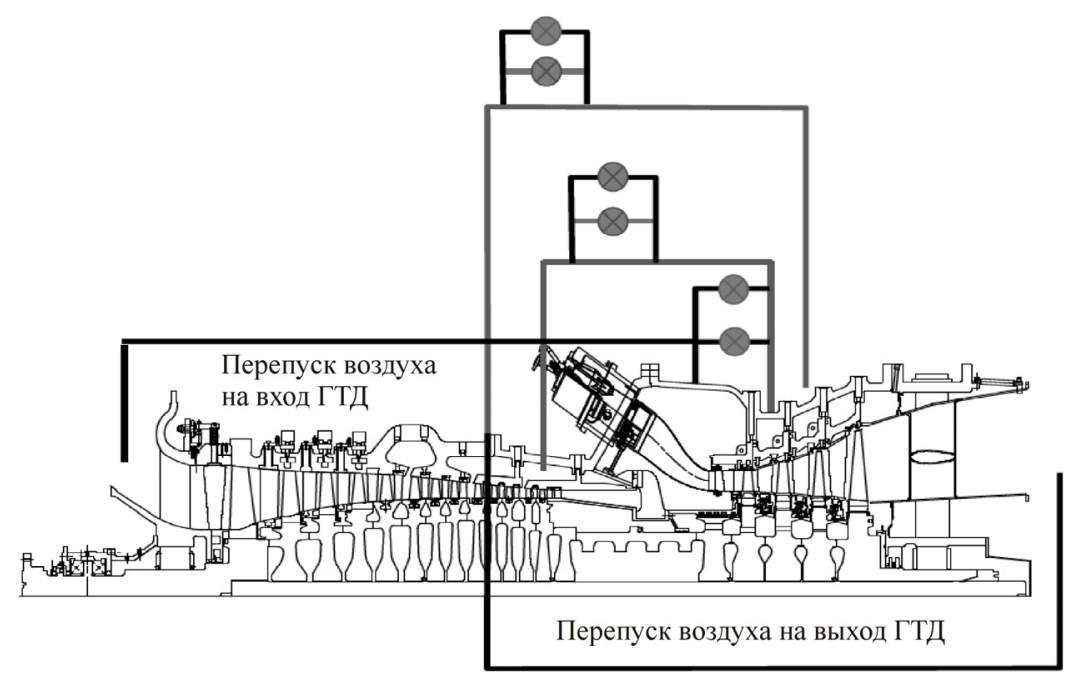

Рис. 7. Схема управления расходом воздуха ГTУ SGT6-5000F

Таким образом, в большинстве ГТУ, эксплуатируемых в настоящее время, для обеспечения работы в малоэмиссионном режиме при изменении параметров ГТУ (за счет дросселирования или внешних условий) поддерживается температура в зоне горения. Это обеспечивается за счет поворотного ВНА и перепуска воздуха либо на вход в двигатель, либо в газосборник, либо в выхлопную шахту. Перепуск воздуха имеет недостаток, связанный с существенным уменьшением перепада давления на стенке жаровой трубы при открытии перепуска, что оказывает влияние на характеристики камеры сгорания, в особенности на охлаждение стенок жаровой трубы [15]. Конкретные цифры о величинах перепуска воздуха в зарубежных ГТУ отсутствуют, однако для поддержания коэффициента избытка воздуха в зоне горения в заданном диапазоне перепуск на вход в двигатель или в выхлопную шахту требует значительно меньшего количества воздуха в сравнении с перепуском в газосборник. Это говорит о том, что с точки зрения поддержания постоянных параметров в камере сгорания и предотвращения ухудшения охлаждения ТВД перепуск на вход в двигатель является более привлекательным решением, чем перепуск в газосборник.

\section{Применение мирового опыта регулирования ГТД с МЭКС}

Основные направления разработки по созданию МЭКС ориентированы (первоначально) на ГТУ мощностью 16 и 25 МВт.

Основной задачей в создании «бедных» МЭКС является возможность гибко управлять профилем концентрации в зоне горения, что позволит обеспечить широкий диапазон работы ГТД с МЭКС без дополнительных средств регулирования. Исходя из этого выбрана четырехколлекторная схема топливопитания. Несомненно, что четырехколлекторная схема топливопитания МЭКС значительно усложняет и удорожает конструкцию ГТД, однако технология гибкого управления полем концентрации в зоне горения является критической для создания малоэмиссионной КС, работающей по «бедному» типу.

Однако даже при возможности гибкого управления профилем концетрации топлива в МЭКС за счет многоколлекторной топливной системы без системы управления регулированием расхода воздуха, поступающего в зону горения, невозможно создать работоспособный во всем климатическом диапазоне $\left(T_{\text {вх }}=(+45 \ldots-60){ }^{\circ} \mathrm{C}\right)$ ГТД с МЭКС, что подтверждается мировым опытом.

Выбор системы управления регулированием расхода воздуха, поступающего в зону горения, осуществлялся на базе решений, широко используемых в зарубежных ГТД, а именно:

- управление поворотом лопаток входного направляющего аппарата компрессора; 
- перепуск воздуха в газосборник;

- отбор воздуха из-за компрессра с дальнейшим его перепуском на вход либо в атмосферу.

Сброс части воздуха (минуя зону горения) в газосборник влечет за собой ряд трудностей с точки зрения технической реализации. Как видно из мирового опыта регулирования ГТД, данный способ практически не используется.

Рассмотрены следующие варианты отбора воздуха за компрессором для поддержания заданного уровня температуры в зоне горения МЭКС:

- с подачей на вход в двигатель;

- со сбросом воздуха в атмосферу.

Наиболее эффективным (с точки зрения минимизации количества отбираемого воздуха) является вариант отбора воздуха из-за компрессора с подачей его на вход в двигатель.

\section{Заключение}

Выполнен анализ мирового опыта регулирования газотурбинных двигателей с малоэмиссионной камерой сгорания по режимам мощности и в климатическом диапазоне. На основании выполненного анализа видно, что критически важно управлять профилем концентрации в зоне горения за счет гибкого управления топливопитанием, что позволит обеспечить широкий диапазон работы ГТД с МЭКС без дополнительных средств регулирования. Однако даже при возможности гибкого управления профилем концентрации топлива в МЭКС за счет многоколлекторной топливной системы без системы управления регулированием расхода воздуха, поступающего в зону горения, невозможно создать работоспособный во всем климатическом диапазоне $\left(T_{\text {вх }}=(+45 \ldots-60){ }^{\circ} \mathrm{C}\right)$ ГТД с МЭКС, что подтверждаетя мировым опытом.

На основании мирового опыта регулирования ГТД с малоэмиссионными камерами сгорания определены основные механизмы регулирования для поддержания постоянной температуры в зоне горения, а именно:

1. Различные способы сброса воздуха из-за компрессора с подачей перепускаемого воздуха на вход в ГТД либо в шахту выхлопа.

2. Перепуск воздуха в газосборник.

3. Управление воздухом за счет поворотных лопаток входного направляющего аппарата компрессора.

\section{Библиографический список}

1. Technology update on gas turbine dual fuel, dry low emission combustion systems / P.E. Rokke, J.E. Hustad, N.A. Rokke, O.B. Svendsgaard // Proceedings of ASME Turbo Expo 2003 Conference. June 16-19. Atlanta, 2003. - Paper № GT2003-38112.

2. Lieuwen T.C., Yang V. Gas turbine emissions. - Cambridge university press, 2013. - 368 p.

3. Лефевр А. Процессы в камерах сгорания ГТД. - М.: Мир, 1986. - 566 с.

4. Boyce M.P. Gas turbine engineering handbook. - 3rd ed. - Elsevier, 2006. - 936 p.

5. Wilis J.D., Moran J.A. Industrial RB211 DLE gas turbine combustion update // Proceedings of ASME Turbo Expo 2000 Conference, May 8-11. - Munich, 2000. - № 2000-GT-109.

6. Rolls-Royce industrial trent: combustion and other technologies / C. Barkey, S. Richards, N. Harrop, P. Kotsiopriftis, R. Mastroberardino, D. Squires, T. Scarinci // Proceedings of International Symposium of Air Breathing Engines. - 1999. - № ISABE 99-7285.

7. Smith K.O., Rawlins D.C., Steele R.C. Developments in dry low emissions systems // Proceedings of 2000 International Pipeline Conference. - Vol. 2. - № IPC2000-267.

8. Ginter T., Crabos O. Upgrade options for the MS6001 heavy-duty gas turbine // GE Reference Document № GER-4217B. - GE Energy, 2010.

9. E-class late fuel staging technology delivers flexibility leap / C.E. Romoser, J. Harper, M.B. Wilson, D.W. Simons, J.V. Citeno, M. Lal // Proceedings of ASME Turbo Expo 2016 Conference. June 13-17. - Seoul, 2016. - № GT2016-57964. 
10. Advanced dry low $\mathrm{NO}_{x}$ combustor for Mitsubishi $\mathrm{G}$ class gas turbines / S. Tanimura, M. Nose, K. Ishizaka, S. Takiguchi // Proceedings of ASME Turbo Expo 2008 Conference. June 9-13. - Berlin, 2008. № GT2008-50819.

11. Development of $1600^{\circ} \mathrm{C}$-class high efficiency gas turbine for power generation applying J-type technology / M. Yuri, J. Masada, K. Tsukagoshi, E. Ito, S. Hada // Mitsubishi Heavy Industries Technical Review. 2013. - September. - Vol. 50, № 3.

12. Design and early development of the SGT-300 twin shaft gas turbine / B.M. Igoe, C. Engelbert, K. Scott, S. Charlton, T. Mapleston // Proceedings of 19th Symposium of the Industrial Application of Gas Turbines Committee. October 17-19. - Canada, 2011. - № 11-IAGT-201.

13. Siemens W501F gas turbine: Ultra low $\mathrm{NO}_{x}$ combustion system development / R. Bland, W. Ryan, K. Abou-Jaoude, R. Bandatu, A. Haris. B. Rising // Proceedengs of POWER-GEN International Conference. - 2004.

14. Foust A. Siemens SGT6-5000F gas turbine technology update // Proceedings of POWER-GEN International Conference. December 8-10. - Las Vegas, 2015.

15. Verification of single digit emission performance of a $24 \mathrm{MW}$ gas turbine - SGT-600 3rd generation DLE / A.M. Carrera, P. Geipel, A. Larsson, R. Magnusson // Proceedings of ASME Turbo Expo 2017 Conference. June 26-30. - Charlotte, 2017. - № GT2017-63089.

\section{References}

1. Rokke P.E., Hustad J.E., Rokke N.A., Svendsgaard O.B. Technology update on gas turbine dual fuel, dry low emission combustion systems. Proceedings of ASME Turbo Expo 2003 conference, paper No. GT200338112, June 16-19, 2003, Atlanta, USA.

2. Lieuwen T.C., Yang V. Gas turbine emissions. Cambridge university press. 2013, 368 p.

3. Lefevre A. Protsessy v kamerakh sgoraniya GTD [Gas turbine combustion]. Moscow: Mir, 1986, 566 p.

4. Boyce M.P. Gas turbine engineering handbook. - 3rd ed. - Elsevier. 2006, 936 p.

5. Wilis J.D., Moran J.A. Industrial RB211 DLE gas turbine combustion update. Proceedings of ASME Turbo Expo 2000 conference, paper No. 2000-GT-109, May 8-11, 2000, Munich, Germany.

6. Barkey C., Richards S., Harrop N., Kotsiopriftis P., Mastroberardino R., Squires D., Scarinci T. Rolls-Royce Industrial Trent: combustion and other technologies. Proceedings of International Symposium of Air Breathing Engines 1999, parer No. ISABE 99-7285.

7. Smith K.O., Rawlins D.C., Steele R.C. Developments in dry low emissions systems. Preceedings of 2000 International pipeline conference, Vol. 2, paper No. IPC2000-267.

8. Ginter T., Crabos O. Upgrade options for the MS6001 heavy-duty gas turbine. GE reference document No. GER-4217B. GE Energy. - 2010.

9. Romoser C.E., Harper J., Wilson M.B., Simons D.W., Citeno J.V., Lal M. E-class late fuel staging technology delivers flexibility leap. Proceedings of ASME Turbo Expo 2016 conference, paper No. GT201657964, June 13-17, 2016, Seoul, South Korea.

10. Tanimura S., Nose M., Ishizaka K., Takiguchi S. Advanced dry low NOx combustor for Mitsubishi G class gas turbines. Proceedings of ASME Turbo Expo 2008 conference, paper No. GT2008-50819, June 9-13, 2008, Berlin, Germany.

11. Yuri M., Masada J., Tsukagoshi K., Ito E., Hada S. Development of $1600^{\circ}$ C-class high efficiency gas turbine for power generation applying J-type technology. Mitsubishi Heavy Industries Technical Review, vol. 50, no. 3, September 2013.

12. Igoe B.M., Engelbert C., Scott K., Charlton S., Mapleston T. Design and early development of the SGT-300 twin shaft gas turbine. Proceedings of 19th Symposium of the industrial application of gas turbines committee, paper No. 11-IAGT-201, October 17-19, 2011, Canada.

13. Bland R., Ryan W., Abou-Jaoude K., Bandatu R., Haris A. B. Rising. Siemens W501F Gas Turbine: Ultra Low $\mathrm{NO}_{x}$ Combustion System Development. Proceedengs of POWER-GEN International conference, 2004.

14. Foust A. Siemens SGT6-5000F Gas turbine technology update. Proceedings of POWER-GEN International conference, Las Vegas, NV, USA, December 8-10, 2015.

15. Carrera A.M., Geipel P., Larsson A., Magnusson R. Verification of single digit emission performance of a 24 MW gas turbine - SGT-600 3rd generation DLE. Proceedings of ASME Turbo Expo 2017 conference, paper No. GT2017-63089, June 26-30, Charlotte, USA. 


\section{Об авторах}

Нугуманов Алексей Дамирович (Пермь, Россия) - аспирант кафедры «Авиационные двигатели» ФГБОУ ВО ПНИПУ (614000, г. Пермь, Комсомольский пр., д. 29), инженер отдела камер сгорания АО «ОДК-Авиадвигатель» (614990, г. Пермь, Комсомольский пр., д. 93, e-mail: nugumanov@avid.ru).

Сипатов Алексей Матвеевич (Пермь, Россия) - доктор технических наук, профессор кафедры «Авиационные двигатели» ФГБОУ ВО ПНИПУ (614000, г. Пермь, Комсомольский пр., д. 29), начальник отдела камер сгорания АО «ОДК-Авиадвигатель» (614990, г. Пермь, Комсомольский пр., д. 93, е-mail: sipatov@avid.ru).

Назукин Владислав Алексеевич (Пермь, Россия) - инженер-конструктор-расчетчик отдела камер сгорания АО «ОДК-Авиадвигатель» (614000, г. Пермь, Комсомольский проспект, д. 93, e-mail: v.a.naz@narod.ru), доцент кафедры «Авиационные двигатели» ФГБОУ ВО ПНИПУ (614000, г. Пермь, Комсомольский пр., д. 29).

\section{About the authors}

Aleksey D. Nugumanov (Perm, Russian Federation) - Postgraduate Student, Aviation Engines Department, Perm National Research Polytechnic University (29, Komsomolsky av., Perm, 614000); Engineer, Combustor Chambers Department, UEC-Aviadvigatel JSC (93, Komsomolsky av., Perm, 614990, e-mail: nugumanov@avid.ru).

Aleksey M. Sipatov (Perm, Russian Federation) - Doctor of Technical Science, Professor, Aviation Engines Department, Perm National Research Polytechnic University (29, Komsomolsky av., Perm, 614000); Chief, Combustor Chambers Department, UEC-Aviadvigatel JSC (93, Komsomolsky av., Perm, 614990, e-mail: sipatov@avid.ru).

Vladislav A. Nazukin (Perm, Russian Federation) - Simulation and Design Engineer, Combustor Chambers Department, UEC-Aviadvigatel JSC (93, Komsomolsky av., Perm, 614990, e-mail: v.a.naz@narod.ru), Associate Professor, Aviation Engines Department (29, Komsomolsky av., Perm, 614000).

Получено 26.07.2017 